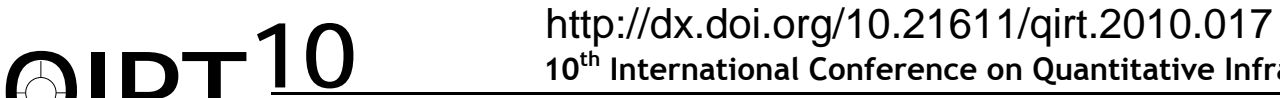 \\ $10^{\text {th }}$ International Conference on Quantitative InfraRed Thermography \\ July 27-30, 2010, Québec (Canada)
}

\section{Continuous monitoring of radiotherapy through infrared image processing}

\author{
by I. Benkö Prof.Dr.techn. and G.J.Köteles Prof.Dr.med. ${ }^{*}$
}

"Corresponding author: Fac.of Mech.Engineering, Budapest University of Technology and

Economics,Budapest,Hungary, H-1112 Budapest, Cirmos u.1., Hungary, Fax/Phone:36-1-310-0999,ibenko@freestart.hu

** 'Frédéric Joliot-Curie' National Research Institute for Radiobiology and Radiohygiene, Budapest,Hungary

Keywords: image processing, medical infrared images, monitoring, radiotherapy

\begin{abstract}
Radiation therapy (radiotherapy) is a valuable technique used in medicine cancer treatment. It is a fact that many of the incidents and accidents involve only partial body irradiation and the extremities are the most frequently injured parts of the body. As it is still not general practice to use infrared (IR) thermographic imaging for the more precise diagnosis of local injuries, the availability of these techniques is very rarely mentioned in the publications. In this paper attention is drawn to what was introduced more than a decade ago, namely the use of IR thermographic procedures in diagnosing local radiation injuries. Further justification for introducing these techniques is that, in the relevant cases, the assesment of absorbed radiation dose through the widely known biological indicators or biological dosimetry assays is not always possible.
\end{abstract}

\section{Introduction: image analysis}

In determining the temperature fields of various characteristics, the choice is between the following general methods, while their relative advantages and disadvantages must be decided in the light of the test being performed:

(a) the selection of the temperature interval to be tested and, within that, the decision over the choice of the number and widths of the isostrips;

(b) determining the temperature at specified points of the surface under test (e.g. the centre point of the cross hairs);

(c) ) comparison between temperature distributions along the horizontal and vertical lines;

(d) determining the temperature distribution and mean temperature in smaller specified areas of the surface tested;

(e) statistical methods for the description of temperature distribution (e.g. histographic processing, and distribution curve of the histogram) and image filtering.

Histographic analysis is the usual mode of processing experimentally and otherwise obtained sets of data, but it may be considered an efficient way for describing temperature fields, too (figures 1 and 2). Thereby there is still little experience available for such applications and for the proper evaluation of all histogram characteristics [1,2,3,4].

In histogram which represent temperature fields of digital infrared(IR) images (figure 1), pixel numbers (in percentages) with the given temperature are plotted against temperatures occuring within the fields (see [5], figures 4 and 5). Temperatures occuring in the selected area may be displayed both graphically and digitally, and the obtained data lend themselves to further computation (figure 2). Satisfactory result were obtained by the present authors when the temperature varied by tenths of degrees within the range.

\section{Generalities}

In medicine, two techniques for thermal imaging can be applied: contact thermography and IR thermogrammetry. Although the former is simplier and the equipment is less expensive, the information provided by the latter is more detailed. The following information can be obtained and this is important for medical application:

(a) the detection of injuries mainly in comparison with the relevant contralateral part of the body,

(b) visualization of the extent of the injury and

(c) the possibility of follow-up of the pathological condition.

Thermographic evidence of previous radiation exposure can also be obtained in the latent phase of the pathological process [2]. Unfortunately, the dose estimation cannot be performed immediately from the thermal map(thermogram). Recently, however, it is considered to be of value to demonstrate the dose range in which the thermographic signal, i.e. the change in the local temperature, indicates the radiation injury or burn. Accordingly, a series of measurements were initiated on patients submitted to ionizing radiation after surgical mastectomy. The evaluation of thermograms was made by computerized analysis transforming the altered temperature distribution to histograms $[5,6]$. By this more sensitive approach to analysis it was found that temperature alterations following even a dose $2.5 \mathrm{~Gy}$ from accelerated electron irradiation after the first treatment session could be detected and followed during the whole 
treatment. In a case report, the present authors also published a successful demonstration of temperature increase following an accidental 1-2 Gy $\mathrm{y}$ irradiation of a hand [2].

\section{Method of examination}

The investigations on the subjects treated with $\beta$ irradiation to the chest were performed using AGA THV 780-type IR imaging equipment $[5,6]$. The images were stored from the different investigations, over 3 weeks by IR video recording, which was evaluated by computerized image analyses.

The method of data analysis was elaborated and applied for the investigation of six patients. The irradiated areas as shown in reference [5] (Fig.1) have absorbed 2.5 Gy at each session but from various energies. The radiation treatment was performed for consecutive weeks, five irradiation sessions per week applied daily; the $\beta$ dose was 12 times $2.5 \mathrm{~Gy}$. The treatment on weekend days were omitted. These post-operative irradiations always complete the complex therapy of breast cancer patients. The immediate effect could be seen within 20-30 min after irradiation.

Among essential values characteristic of the histograms, the following are selected: the highest (maximum), the lowest (minimum) and the average temperature in the defined area; the median, standard deviation and skewness; the number of pixels in the examined area and the maximum value on the ordinate of the histogram.

The distribution curve $D$ of the histogram is the integral of the histogram (figure 1 ) and the derivative $\mathrm{d} D / \mathrm{dt}$ of the distribution curve gives the shape of the histogram (figure 2). The series of distributions of histograms (figure 3 ) is more visual and efficient for comparisons than a series of histograms (figure 1). For illustration, figures 1 to 3 are taken from the effects of $\beta$ irradiation.

\section{Analysis of the effects of radiation treatmment}

Following the post-operative radiation treatment over a period of 3 weeks, three phases of effect were observed. The following characteristics of the thermal reactions were identified : in the case of the thermal approach to the quantitative measurement of radiodermatitis of ionizing irradiation, the thermal skin reactions at the beginning affected by the thermal equilibrium of two main factors, namely biological phenomena of irradiation effects and thermal regulation, i.e. injury and tissue regeneration.

The dynamics of the thermal reactions in the first week can be seen in figure 3. During the first week the patient was irradiated on consecutive days, four times, with $2.5 \mathrm{~Gy}$ each session. It is obvious that, after the first treatment session, i.e. $2.5 \mathrm{~Gy}$, the temperature has been considerably increased. The value of the median temperature changed approximately 32.2 to $34.5^{\circ} \mathrm{C}$. The shape of the distribution curve was not significantly changed.

Accepting that the increased temperature is a normal tissue reaction to radiation-induced skin reaction(figure 3 ), it can be seen that at the end of the first session the skin temperature increased (figure 3) in contrast with the second and third sessions (weekends) when it was decreased (figures 4 and 5). This may indicate that the regulatory functions of the skin have been relaxed. On further irradiation, the radiation reaction were not additive; just a decrease of the average temperature was observed especially when there was a break of two days in the treatment protocol. Accordingly, the increases in radiation doses do not increase the tissue reactions but, in contrast, induce a modulation.

\section{Conclusion}

Lacking reliable dosimetry, the radiotherapist must consider the nature and severity of signs and symptoms, the protracted expression of the injury, and the timing and differential expression of injury in various tissues, when deciding treatment options and prognosis.

In summary, the measurements from the thermal image proved to be useful tools in both the prognosis of local radiation injuries in a wide dose range. A definitive non-invasive method to determine the extent and magnitude of a local or partial body radiation injury was found [7].

In addition, numerous techniques can be utilized to evaluate circulation in an affected area, to determine the volume, depth and area of tissue affected. Other medical imaging techniques include angiography, radionuclide imaging and non-invasive technique such as impedance plethysmography, magnetic resonance imaging and ultrasound. Techniques capable of evaluating superficial blood flow and tissue perfusion have clinical value for the physician, who must counsel the patient and make critical decisions regarding medical or surgical treatment. 


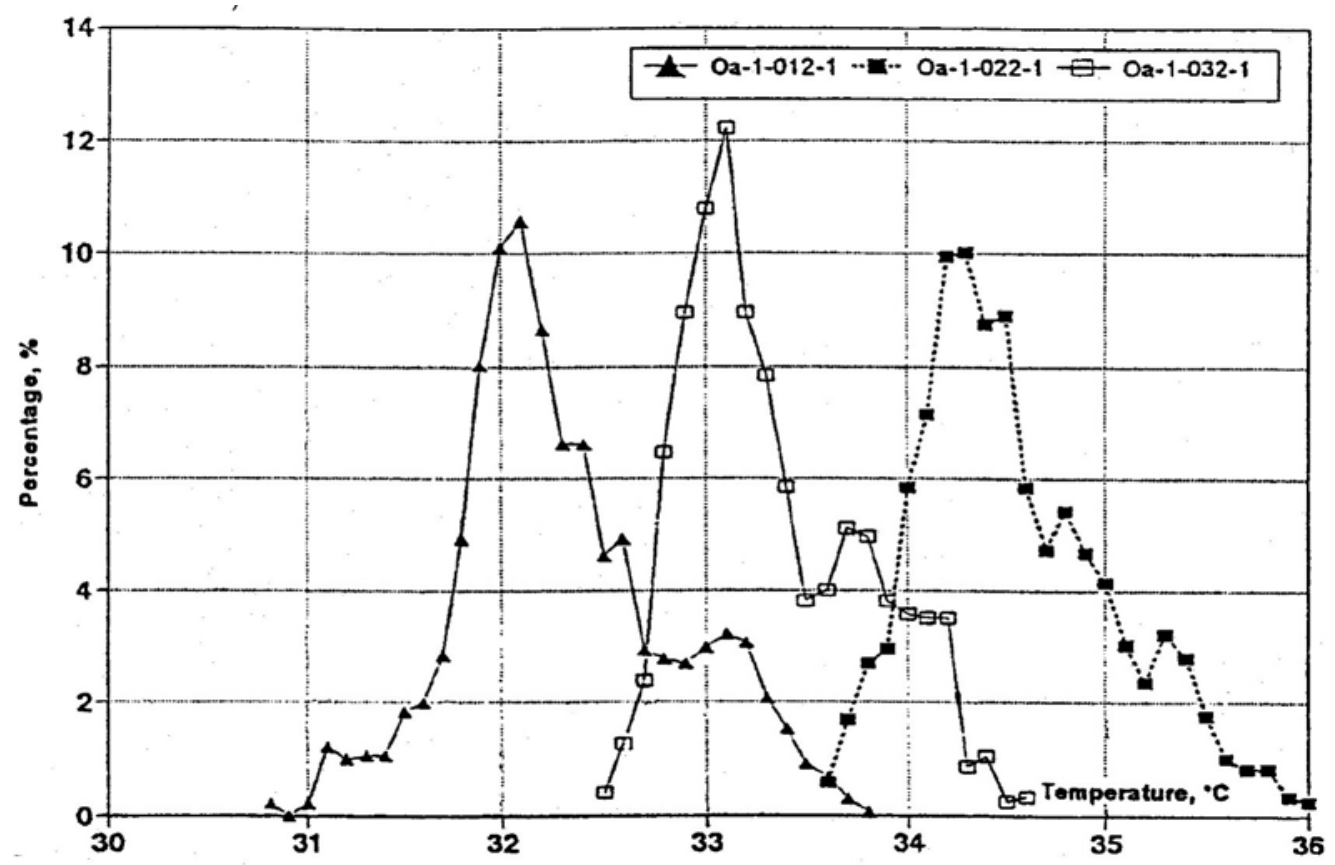

Fig. 1. Series of histograms of the temperature values on the $\beta$-irradiated areas on the first $(-\mathbf{-})$, second ( $\cdot \cdot)$ and third ( $\square)$ day during radiation treatment (first week)

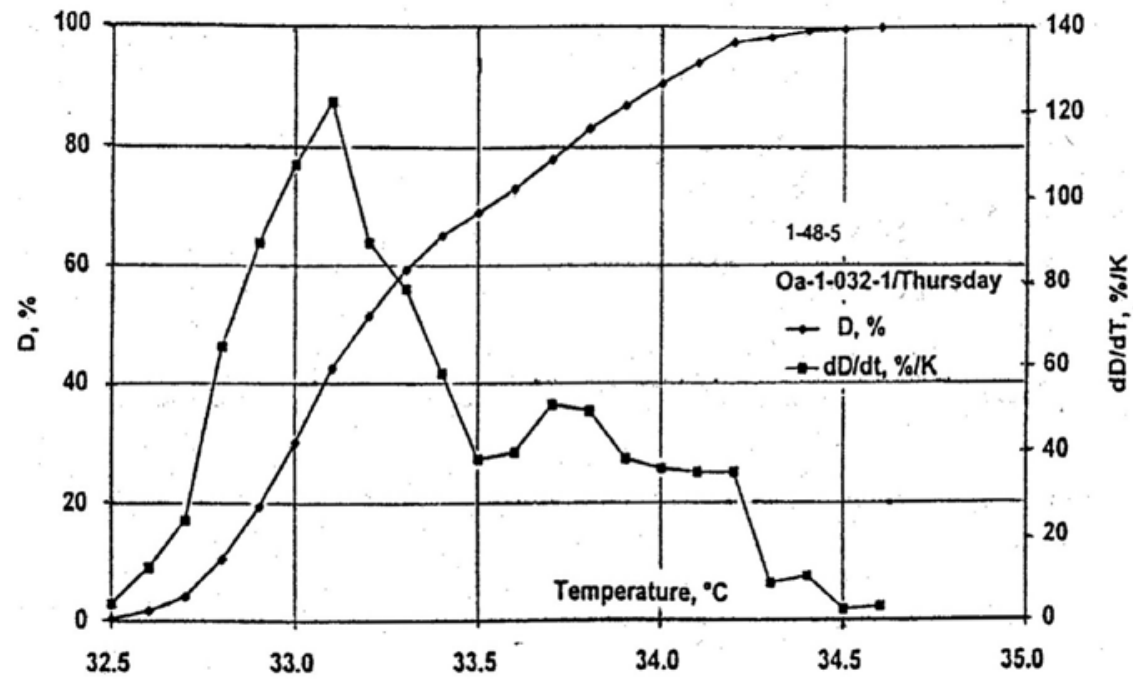

Fig. 2. The distribution of a temperature histogram $D$ and its derivative $d D / d t$, i.e. the shape of the histogram (after the second session) 


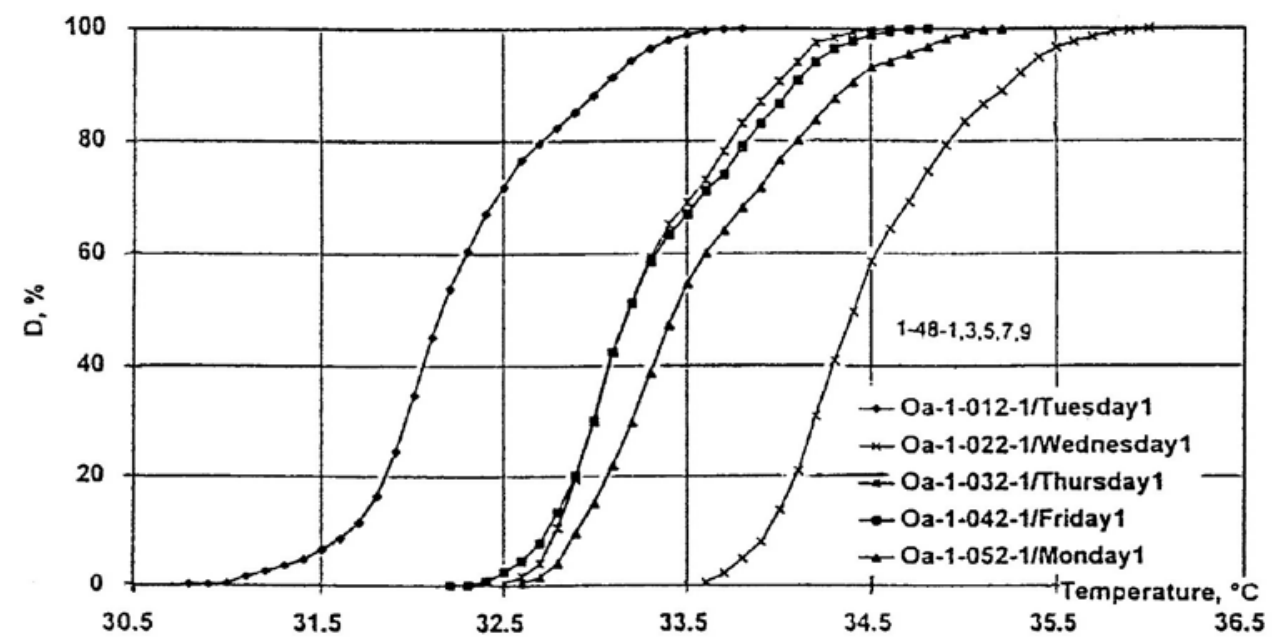

Fig. 3. Series of distributions of the temperature histograms of $\beta$-irradiated areas in the first week

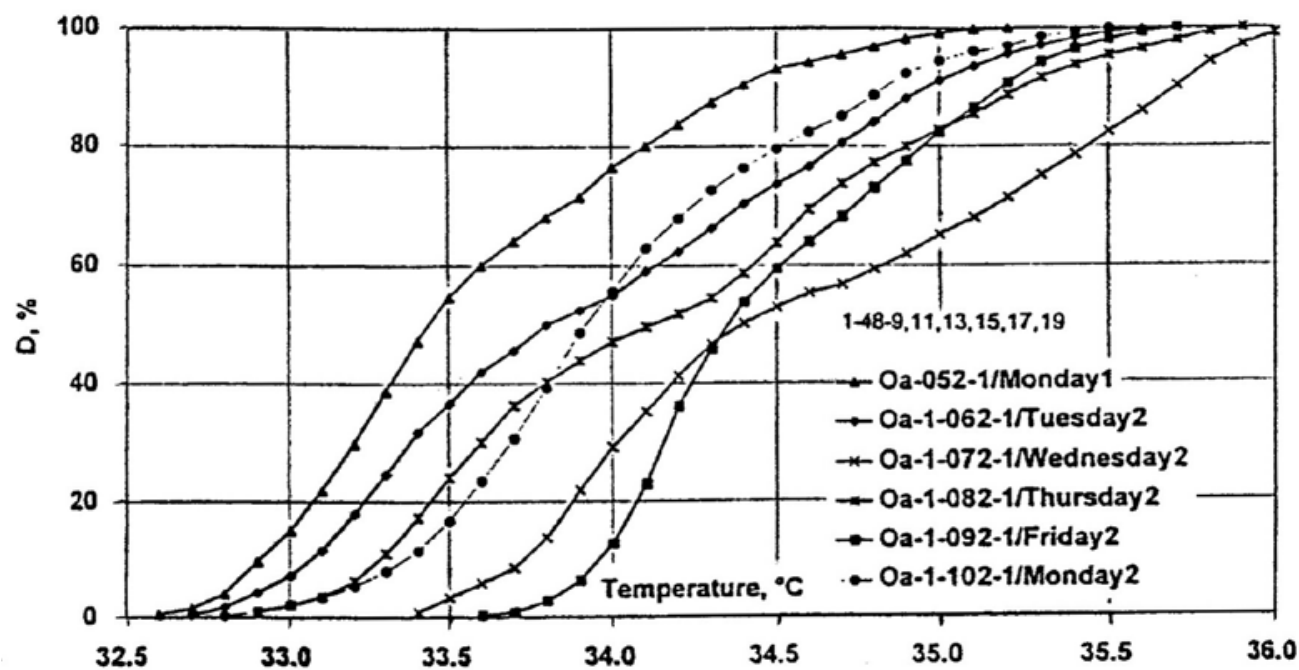

Fig. 4. Series of distributions of the temperature histograms of $\beta$-irradiated areas in the second week 


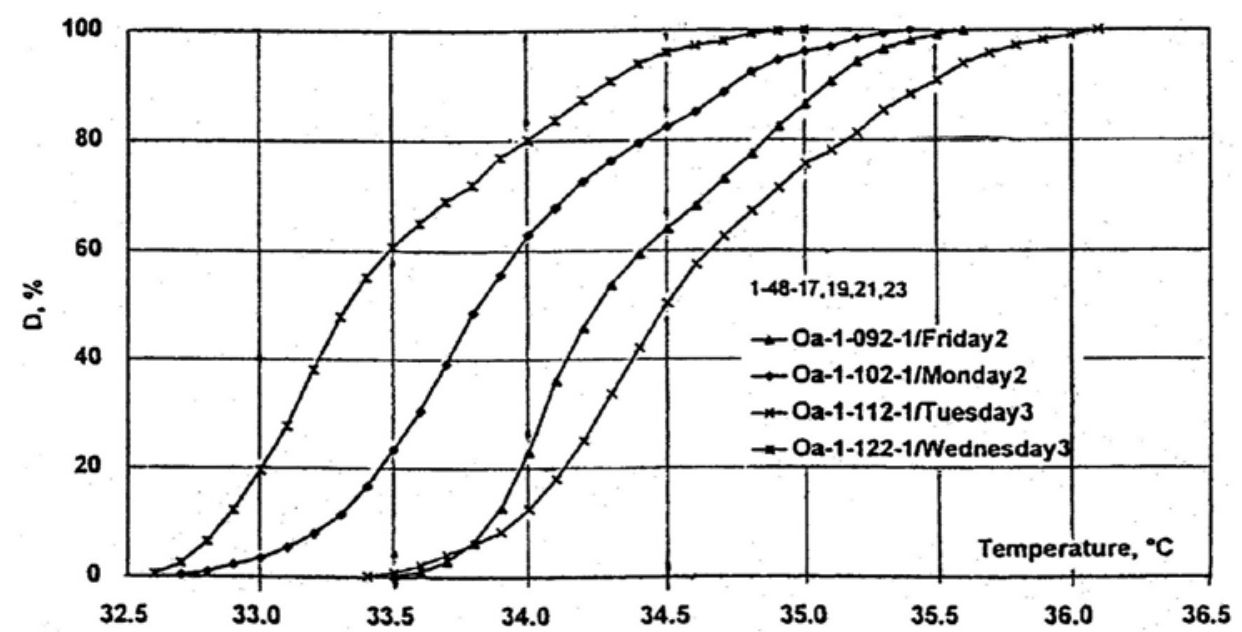

Fig. 5. Series of distributions of the temperature histograms of $\beta$-irradiated areas in the third week

\section{REFERENCES}

[1] Benkö, I. Quantitative analysis of medical infraredpictures.Thermol.Öst.,August,2,17(1992).

[2] Benkö, I. and Köteles, G.J. Thermotechnical approaches to the investigation of local injurie caused by ionising radiation. Periodica Polytechnica, Ser. Mech. Engng, 37, pp.197213(1993).

[3] Benkö, I. Histographical analysis of infrared images for medical diagnosis. In Proceedings of Eighth International Conference on Thermal Engineering and Thermogrammetry, (Ed. I. Benkö),pp.307-308,Budapest,Hungary (1993).

[4] Benkö,l. Possibilities of infrared imagery in the field of biology of ionising radiation. In Proceedings of Workshop on Advanced Infrared Technology and Application, Casa Malaparte Capri, Italy, 20-21 September 1993, pp.255-270 (Fondazione 'Giorgio Ronchi'), Firenze (1994).

[5] Benkö, I. ; Köteles, G.J. and Németh, G. Thermal imaging of the effects of beta-irradiation on human body surface. In Proceeding of the Conference on Quantitative Infrared Thermography (QIRT '96), Eurotherm Series 50, Stuttgart,Germany, 2-5 September 1996, pp.354-359(Edizioni ETS)Pisa (1996).

[6] Benkö, I. and Köteles, G.J. Recent data concerning human reaction against ionizing radiation. In Proceedings of the Conference on Recent Advances in Medical Thermology. In Thermol.Öst., 4, pp.181-182 (1994).

[7] Benkö, I. ; Köteles, G.J. and Németh, G. Use of thermogrammetry in diagnosis of localradiation injuries. Health Physics, 74, pp.264-265 (1998). 